\section{P68 WHAT IS THE EVIDENCE FOR INCREASED CARDIOVASCULAR DISEASE RISK IN CHILDREN AND YOUNG PEOPLE WITH EMOTIONAL MENTAL HEALTH DISORDERS?}

A Zapiti, LD Hudson*. Institute of Child Health, University College London, London, UK

10.1136/bmjpo-2019-RCPCH-SAHM.70

Aims Contemporary pathological processes leading to cardiovascular disease (CVD) are known to begin in childhood and young people (CYP), and this can be increased in a number of disease states. It is well established that mental health disorders in adults are strongly linked to CVD with increased associated mortality. Less is known about the associations between CVD risk and mental health disorders in CYP; and it is unclear whether CYP with mental health disorders have higher rates of conventional, detectable CVD risk factors (such as dyslipidaemia), or contemporary pathological processes (such as arterial stiffening). Furthermore, this important question has never been systematically reviewed. We hypothesized that CYP with emotional mental health disorders (depression or anxiety) would have higher rates of CVD risk.

Methods We performed a systematic review of the literature using PRISMA guidelines. PubMed, PsychINFO, Web of Science and Embase were searched from inception to May 2019. Searches identified papers studying associations between CVD risk (contemporary CVD risk markers such as blood pressure, lipids, insulin resistance, central adiposity; established measures of arterial stiffening; or later disease outcomes) and common emotional mental health disorders (depression or anxiety) in CYP. Studies examining obesity and mental health disorders alone were excluded.

Results Results will be presented in narrative format, separated by individual risks and mental health disorder.

\section{\begin{tabular}{|l|l}
\hline P69 INQUIRY OF SOCIAL MEDIA USE IN ADOLESCENTS \\
\hline
\end{tabular} ASSESSED IN A CAMHS CLINIC IN UK}

C James, V Shetty*. Lenworth Clinic (Ashford Child and Adolescent Mental Health Service), North East London NHS FT, Ashford, UK

\subsection{6/bmjpo-2019-RCPCH-SAHM.71}

Aims The Royal College of Paediatrics and Child Health (RCPCH) published guidelines (in 2019) for parents and clinicians on screen time for children and young people. The impact of social media use on a young person's physical and mental health is currently being researched. We are aware of the positive and negative effects of social media use on mental health. Negative effects can include body-image disturbances, cyberbullying, poor sleep, mood disturbances and anxiety.

The aim of the audit was to investigate whether social media usage was considered during an initial assessment (and risk assessment) of a young person in a CAMHS clinic in UK. Methods An Audit was completed at 3 different CAMHS clinics over a 2 month period for all initial assessments. Electronic case records (nationally used) were checked. Total number of cases reviewed were 150 . The authors devised a data collection tool to check for evidence of questions asked in relation to social media usage.

A survey of all CAMHS professionals in the region was also completed to enquire if they routinely asked questions in relation to screen time and social media use during initial assessment.
Results The audit showed less than 5\% of young people assessed in clinics over a 3 month period were routinely asked about their social media use during their initial assessment. Moreover, there was no proportional increase in enquiry for 'high risk' cases. The results are likely to be applicable County-wide as a single electronic record system is used.

The survey showed that social media use and associated risks were not routinely enquired. Furthermore, there were no prompts on the electronic assessment tools for professionals. Conclusions The audit demonstrated a significantly low (almost negligent) percentage of young people (or their parents) actively asked about social media use and its impact on their mental health. Given the likely effects especially in a vulnerable population, it is important these are asked routinely.

The authors devised a simple screening tool to be part of the assessment tool on the electronic records.

Local teaching sessions were also arranged with clinicians and parent workshops were organised.

A re-audit will be completed to evaluate the impact of the training and workshops.

\section{P71 FATAL ACCIDENTS OF CHILDREN AND ADOLESCENTS DRIVING TRACTORS IN RURAL ICELAND}

J Einarsdóttir*, G Gunnlaugsson. Sociology, Anthropology, and Folkloristics, University of Iceland, Reykjavik, Iceland

\subsection{6/bmjpo-2019-RCPCH-SAHM.72}

Aims Across historical periods and societies, capacity attributed to children varies. A case in point is changing views on the appropriate age of children driving tractors off-roads in Iceland where their use exploded after the World War II. Farmers had little experience in driving vehicles, and children were trusted with the task. Here we describe and analyse tractor driving of urban children who stayed on farms in Iceland during the summer, and evaluate the extent of fatal tractor accidents by age and gender.

Method Qualitative study that uses secondary data, including reports from the Administration of Occupational Safety and Health and news reports of fatal tractor accidents, and stories of individuals who were sent to stay at farms during the summer as children from the period 1950 to 2018. This is complemented with quantitative data from a representative survey data on adults who stayed on farms in childhood.

Results Stories expose adolescents' fascination with the adventurous experience of driving tractors, and about half of children who had stayed on farms in childhood had driven tractors; for boys the mean age for first driving was 11.0 years compared to 12.6 years for girls. In total 46 children (85\% boys) died in a tractor accident in the period at the mean age of 10.5 years (median 11.5, range 1-17); $23(50 \%)$ were 12-17 years of age while driving the tractor. In the period 1959-1987, when there was no age-limit for driving tractor, 1.6 out 100,000 children died in a tractor accidents compared to 0.3 in earlier and later periods.

Conclusions Driving tractors during stays on farms caused many fatalities, in particular among adolescents driving tractors. The experience of children driving tractors in Iceland exposes the diverse views on children's capacity. Preventive 